\title{
Acute Rheumatic Endocarditis
}

National Cancer Institute

\section{Source}

National Cancer Institute. Acute Rheumatic Endocarditis. NCI Thesaurus. Code C35201.

Inflammation of the endocardium in acute rheumatic heart disease. 